P105 (continued)

of participants consumed all of the box's contents, increasing their nutrition.

Conclusion: The high percentage of WCK food boxes completely consumed suggests that boxes offering fruits and vegetables are an effective intervention in improving nutrition for recipients. These findings have important implications for shaping nutritional guidance of future food box programs.

Funding: None.

\section{P106 Evidence-Based Extension Education: Self- Reported Knowledge, Practice, and Attitudes of Nutrition Educators}

Alison O'Donoughue, MS, University of Florida; Wendy Dahl,PhD, RD, wdahl@ufl.edu, University of Florida, 359

FSHN Building 572 Newell Dr, Gainesville, FL, 32611

Background: Excellence in nutrition education requires a strong base in research evidence. While evidence-based practice is recognized as essential for nutrition education programming, little research in this area has targeted nutrition educators.

Objective: The aim of the study was to evaluate the perceived knowledge, skills, abilities, attitudes, and activities of nutrition extension educators related to evidence-based practice.

Study Design, Settings, Participant: Family and consumer sciences (FCS) extension agents in Florida were surveyed using an online, modified version of the Evidence-based Practice Questionnaire (EBPQ) through Qualtrics.

Measurable Outcomes/Analysis: Evidence-based practice activities over the past year $(1=$ never to $7=$ frequently), attitudes towards evidence-based practice ( $1=$ very negative to $7=$ very positive agreement), and knowledge, skills and abilities related to evidence-based practice $(1=$ poor to $5=$ excellent $)$ were rated. Preferred sources of evidence-based nutrition information also were queried.

Results: Of the 85 nutrition educators surveyed, 20 FCS agents completed the survey. Regarding undertaking evidence-based practice activities to address gaps in knowledge, participants reported a rating of $5.2 \pm 1.5$ $($ mean $\pm S D)$. Attitudes were rated at $5.7 \pm 1.5$ and perceived knowledge, skills and abilities averaged 3.6 \pm 0.8 . Sources of evidence-based information included professional organizations (American Diabetes Association, American Heart Association, Academy of Nutrition and Dietetics, and the Society of Nutrition Education and Behavior), government departments (U.S. Department of Agriculture), university-approved curriculum and online resources, reputable domain names (.edu, .org, and .gov), nutrition journals, and state extension specialists.

Conclusion: FCS extension agents reported applying evidence-based practice activities to support their nutrition extension programming and very positive attitudes towards evidence-based nutrition education. However, lower ratings of perceived knowledge, skills and abilities suggest a need for professional development opportunities related to evidence-based practice.

Funding: None.

\section{P107 Predictors of Food and Water Stockpiling During the COVID-19 Pandemic}

Olufemi Fabusoro, MS, RD, University of Illinois at UrbanaChampaign; Chelsea Singleton, PhD, University of Illinois at Urbana-Champaign; Margarita Teran-Garcia, PhD, MD, University of Illinois at Urbana-Champaign; Sandraluz Lara-Cinisomo, PhD, laracini@illinois.edu, University of Illinois at Urbana-Champaign, 505 E Armory Ave, Champaign, IL, 61820

Background: The early months of the COVID-19 pandemic brought about significant disruptions in food supply chains, which increased consumers' concern about possible food shortages and price gouging. To ensure personal food security, many consumers began stockpiling food and water in unusually large amounts.

Objective: The goal of this study was to investigate individual- and household-level predictors of food and water stockpiling (FWS) in the early months of the COVID-19 pandemic among Non-Latino Black and Latino adults.

Study Design, Settings, and Participants: This study was a secondary analysis of cross-sectional survey data. Participants were 2,174 Non-Latino Black (66.4\%) and Latino (33.6\%) adults residing in a Midwestern state, who completed the survey in either May or June/July 2020.

Measurable Outcome/Analysis: Participants were asked to self-report (yes or no) if they stockpiled food and/or water in the prior 7 days in response to the pandemic. A variety of variables was examined, including education level, annual income, employment status, concerns about COVID19, and self-quarantine status. Crude and adjusted logistic regressions were used to identify variables associated with FWS. Results: Non-Latino Black participants had lower odds of reporting FWS compared to Latinos (OR 0.64; 95\% CI, 0.51-0.79). Similarly, participants who were not concerned about COVID-19 had lower odds of FWS compared with those extremely concerned (OR 0.37; 95\% CI, $0.20-$ 0.71 ). In contrast, odds of FWS were higher among participants who were self-quarantining all the time compared to those who were not (OR 2.16; 95\% CI, 1.31 - 3.59).

Conclusion: Results showed that Latinos, adults concerned about COVID-19, and self-quarantine status had significantly higher odds of FWS during the pandemic.

Funding: None.

\section{P108 Representation of Older Adults in Nutrition Education and Behavior Published Literature}

Samantha Epstein, University of Florida; Wendy Dahl, PhD, RDFDC, wdahl@ufl.edu, University of Florida, 359 FSHN Building 572 Newell Dr, Gainesville, FL, 32611 


\section{P108 (continued)}

Background: In 2019, the Society of Nutrition Education and Behavior recommitted to representing the growing demographic of older adults. One metric to assess this commitment may be an equitable representation of articles published in the Society's journal.

Objective: The aim of this retrospective study was to determine the historic representation of the older adult life stage in articles published in the Journal of Nutrition Education and Behavior (JNEB).

Study Design, Setting, Participants: Research, research briefs, and report articles published in JNEB from 1969 to December 2020 were examined to determine the targeted life stage, including older adults ( $>65$ years), adults, young adults (college-age), and infants, children and adolescents. Articles, in which older adults were included as part of a greater population, were also quantified. An "other" category included articles that did not target or state a life stage. Measurable Outcomes/Analysis: The percentage of research articles, research briefs and reports targeting the older adult life stage published in JNEB.

Results: Of the 2,454 articles reviewed, 39\% of research articles, $51 \%$ of research briefs, and $29 \%$ of reports targeted infants, children and adolescents. Articles specifically addressing older adults had the least representation and included only $4 \%$ of research articles, $3 \%$ of research briefs, and $4 \%$ of reports. Older adults as part of a greater population, were represented by $8 \%$ of research articles, $9 \%$ of research briefs, and $5 \%$ of reports. Of note, $37 \%$ of articles fell into the "other" category.

Conclusion: Historically, the older adult life stage is underrepresented in articles published in JNEB. Given the Society's recent resolution to increase the focus on the educational needs of a growing aging population, an increase in journal publications targeting older adults is warranted, and thus equitably represent all life stages in future issues.

Funding: None.

\section{P109 Responsive Feeding During COVID-19: Evaluation of a Specialized Training for ECE Providers and Stakeholders.}

DiptiA.Dev,PhD, ddev2@unl.edu, University of NebraskaLincoln, 255 Louise Pound Hall, 512 N 12 St, Lincoln, NE, 68588; Saima Hasnin, MS, University of Nebraska-Lincoln; Madeleine Sigman-Grant, PhD, RD, University of Nevada Cooperative Extension; Janice Fletcher, PhD, University of Idaho; Jill Cox, RD, MS, Penn State Extension

Background: Practicing family style meal service in early care and education (ECE) settings supports children's autonomy and improves self-regulation of energy intake. However, during COVID-19, CDC passed a directive to ECE to pause family style meal service. Therefore, we conducted an ECE webinar focused on how to feed children responsively during plated meal service to help providers support children's autonomy and self-regulation.

Objective: To evaluate changes in the level of understanding, behavioral intention to implement knowledge, and confidence about how to feed children (2-5 years) responsively during plated meal service. Also, to determine need for future training opportunities.

Study Design, Setting, Participants: Retrospective pretest-posttest study. The webinar was advertised through an email newsletter disseminated by a nationallevel platform, Penn State Extension Better Kid Care. Participants attended the online webinar for 1 hour on August 11, 2020, had access to an Extension publication (http://bit.ly/3pnJ71X) and responded to a follow-up survey (Qualtrics Link: http://bit.ly/3jMWvvv). Participants identified themselves as either ECE providers $(n=77)$ or other stakeholders $(n=30)$, who can offer opportunities or guidance to providers.

Measurable Outcome/Analysis: Change in self-reported evaluation score (range $1=$ low to $7=$ high) for understanding, behavioral intention to implement knowledge, and confidence about how to feed children responsively during plated meal service. Paired sample $t$ tests (alpha $=0.05)$ followed by Sidak-Bonferroni correction (adjusted $P=0.007$ ).

Results: Both providers and stakeholders reported significantly higher $(P<0.001)$ level of understanding, intention to implement knowledge obtained, and confidence regarding implementing responsive feeding during plated meal service after the webinar compared to before the webinar. Participants expressed need for online nutrition trainings with emerging themes such as feeding infants, safe food preparation and storage, flexibility of CACFP rules, and physical distance during meals.

Conclusion: Intentional mealtime conversations, giving children small tasks supporting their autonomy, and setting clear expectations while adjusting mealtime routines can offer a promising avenue for implementing responsive feeding during plated meal service.

Funding: None.

\section{P110 The Healthy Communities Initiative: Gathering Community Input During COVID-19}

Jessica Stroope, MPH, jstroope@agcenter.lsu.edu, Louisiana State University AgCenter, 202G Knapp Hall, Baton Rouge, LA, 70803; Matthew Greene, LDN, MSPH, RD, Louisiana State University AgCenter; Katherine Seal, MPH, Louisiana State University AgCenter; Denise Holston, PhD, $L D N, R D N$, Louisiana State University AgCenter

Background: The Louisiana Healthy Communities Initiative, led by the LSU AgCenter, guides SNAP-Ed policy, systems, and environmental change efforts. Prior to COVID19, agents held in-person community forums to gather input and community priorities for interventions. COVID19 spurred innovation in this process. Although many states have used videoconferencing services to host meetings, lack of functional internet access posed a barrier for many communities in Louisiana.

Objective: To gather broad community input through Qualtrics surveys in order to assess needs and assets, guide project prioritization, and gather feedback on previous projects.

Continued on page 576 